\title{
La perdiz roja (Alectoris rufa) en España: especie cinegética y amenazada
}

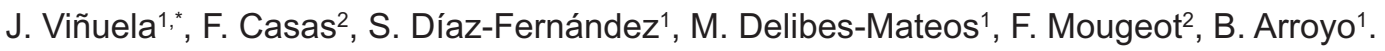 \\ (1) Instituto de Investigación en Recursos Cinegéticos, IREC (CSIC-UCLM-JCCM). Ronda de Toledo s/n 13071, Ciudad Real, España. \\ (2) Estación Experimental de Zonas Áridas (EEZA-CSIC), Carretera de Sacramento, La Cañada de San urbano, 04120 Almería, España.
}

* Autor de correspondencia: Javier Viñuela [Javier.vinuela@uclm.es]

> Recibido el 14 de febrero de 2013, aceptado el 26 de mayo de 2013.

Viñuela, J., Casas, F., Díaz-Fernández, S., Delibes-Mateos, M., Mougeot, F., Arroyo, B. (2013). La perdiz roja (Alectoris rufa) en España: especie cinegética y amenazada. Ecosistemas 22(2):6-12. Doi.: 10.7818/ECOS.2013.22-2.02

La perdiz roja (Alectoris rufa) en España: especie cinegética y amenazada.

Recopilamos el conocimiento científico actual sobre la ecología y gestión cinegética de la perdiz roja, haciendo hincapié en los hallazgos de los últimos años. La perdiz roja es una especie cinegética muy apreciada, cuyo estatus de conservación ha sido, y probablemente es, delicado. En los cotos de caza menor se dedican grandes esfuerzos a su gestión, pero muchas prácticas se aplican sin conocer las consecuencias que tienen para las poblaciones de perdiz. Los estudios revisados indican que el modelo de gestión más adecuado para aumentar la abundancia de esta especie, manteniendo la integridad biológica de sus poblaciones al tiempo que se cazan, incluye un ajuste apropiado de la presión cinegética a la abundancia anual de perdices, el manejo del hábitat promoviendo la vegetación natural en la matriz agrícola y la presencia de lindes, y el aporte suplementario de agua y comida. La información sobre la eficacia del control legal de depredadores generalistas para incrementar la abundancia de perdiz no es concluyente. Las sueltas de perdices de granja ponen en riesgo la integridad genética de la especie. Además, cuando se sueltan en pequeñas cantidades, no aumentan las bolsas de caza ni la rentabilidad económica de los cotos, pero afectan negativamente a la productividad de las poblaciones silvestres de perdices. A pesar del ocio y movimiento económico que genera la caza de perdiz roja, una gestión inadecuada reduce los beneficios potenciales de la actividad.

Palabras clave: control de depredadores, gestión agraria, presión de caza, sueltas

Viñuela, J., Casas, F., Díaz-Fernández, S., Delibes-Mateos, M., Mougeot, F., Arroyo, B. (2013). The red-legged partridge (Alectoris rufa) in Spain: a threatened game species. Ecosistemas 22(2):6-12. Doi.: 10.7818/ECOS.2013.22-2.02

We review scientific knowledge on red-legged partridge ecology and management, focusing on recent studies. Red-legged partridge is a greatly valued game species, whose conservation status has been, and probably still is, poor. In small-game hunting estates, great efforts are devoted to red-legged partridge management, but many practices are applied without knowing their real consequences for partridge populations. Reviewed studies show that the most successful management options to increase the abundance of the species, allowing a sustainable harvest, would include an adequate adjustment of annual harvest to partridge abundance, appropriate habitat management promoting the presence of natural vegetation within the farmland matrix and field edges, and water and food supplementation. Information about the efficacy of predator control to increase red-legged partridge abundance is inconclusive. Releases of farm-reared partridges are putting at risk the genetic integrity of the species. Furthermore, when few partridges are released, releases do not increase annual harvest or estate profitability, and negatively affect the productivity of wild red-legged partridges. Despite leisure and economic movement generated by red-legged partridge hunting, inadequate management is currently reducing the potential benefits of this activity.

Keywords: agrarian management, hunting pressure, predator control, releases

\section{Introducción}

En la literatura cinegética no es difícil encontrar a la perdiz roja proclamada como "la reina" de la caza menor en España. Ciertamente, el origen de la afición por la caza de la Galliforme ibérica más típica se pierde en la antigüedad y la perdiz roja sigue siendo pieza cinegética clave en la actualidad, con 2-3 millones de ejemplares silvestres abatidos anualmente en España en los últimos años, cifras superadas tan solo por el conejo de monte (Oryctolagus cuniculus) (Garrido 2012). Paradójicamente, la perdiz roja estaba considerada especie Vulnerable a nivel europeo (Aebischer y Potts 1994), aunque en los listados más recientes de la UICN (Unión Internacional para la Conservación de la Naturaleza) o Bir- dlife International no aparece ya como especie amenazada. Este cambio de status posiblemente refleja más una disparidad o cambio de criterios que una mejora real del estatus de la especie, ya que según el programa de seguimiento de aves comunes de SEO/Birdlife, la perdiz roja es una de las 10 especies que ha sufrido mayor disminución de abundancia en los últimos años (un $16.2 \%$ desde 1998 a 2012; Seo/Birdlife 2013). ¿Cómo es posible que se cacen anualmente millones de ejemplares de una especie en declive que se ha considerado Vulnerable? ¿Cómo se ha llegado a esta peculiar situación? España tiene un papel primordial en la conservación de la perdiz roja, ya que su área de distribución natural está actualmente restringida a la Península Ibérica, sur de Francia y noroeste de Italia, donde las poblaciones silvestres han sufrido fuertes de- 
clives numéricos, en especial a partir de los años 70-80 (Aebischer y Potts 1994). Además, la perdiz roja desapareció de amplias áreas más norteñas en los dos últimos siglos (originalmente se distribuía hasta el sur de Alemania). Las poblaciones españolas han podido sufrir una disminución superior al 50 \% desde 1973 (Blanco-Aguiar et al. 2003).

El sector cinegético dedica notables esfuerzos para gestionar esta especie en miles de cotos de toda la geografía hispana, proporcionando agua y alimento suplementarios, gestionando el hábitat, controlando la abundancia de sus depredadores, estableciendo cupos de capturas y soltando millones de ejemplares criados en granjas (Arroyo et al. 2012). Sin embargo, la información científica disponible sobre cuáles son los efectos de estos diversos sistemas de gestión sobre las poblaciones silvestres de perdiz roja es muy escasa, y la gestión se basa a menudo más en opiniones, experiencias personales y tradiciones que en criterios técnicos con fundamento científico. Aunque en el mundo cinegético sí parece haber bastante unanimidad sobre el papel de los depredadores: son considerados uno de los problemas más graves para la perdiz roja (Delibes-Mateos et al. 2013a). ¿Es esta una visión realista?

El conocimiento científico sobre esta especie ha mejorado sustancialmente en épocas recientes: por ejemplo, sólo en el IREC se han producido seis tesis doctorales sobre la perdiz roja desde su inauguración en 1999, y se ha publicado un buen número de artículos científicos sobre esta especie. Hoy día sabemos bastante más sobre la realidad de la perdiz roja en España, y trataremos de resumir este conocimiento en las siguientes páginas.

\section{Aspectos ecológicos importantes para la gestión de la perdiz}

\section{Importancia del éxito de nidificación en la demografía}

La perdiz roja es una especie con alta productividad potencial, ya que no sólo tiene un tamaño medio de puesta muy elevado, sino que exhibe un peculiar comportamiento reproductivo de doble puesta: aproximadamente el $50 \%$ de las hembras en años de climatología favorable pone huevos en dos nidos, uno incubado por ella misma y otro por el macho (Green 1984, Casas et al. 2009). La regulación de este peculiar sistema reproductivo parece depender de la condición física de las hembras (Casas et al. 2009) y la depredación (Green 1984). En los casos de doble puesta, el macho contribuye más a la productividad total de una pareja porque el número de huevos incubados por él es mayor y además sufre menos pérdidas por depredación. Estos resultados también indican que la muerte de machos en la caza con reclamo puede tener un impacto demográfico importante.

Por otra parte, la productividad anual de una población de perdiz es altamente dependiente de la climatología (Lucio 1990, Casas et al. 2009), por lo que la abundancia post-reproductora puede variar fuertemente entre años.

\section{Hábitat y gestión agraria}

La perdiz roja es una especie muy adaptable, presente en un amplio rango de hábitats en la mayor parte del país, evitando tan solo la alta montaña, el clima de carácter más atlántico, y los bosques extensos y continuos (Blanco-Aguiar y col. 2003). Los cambios a gran escala en la estructura del hábitat de zonas serranas ocurridos en el último siglo (desaparición de pastizales y cultivos de montaña sustituidos por matorral) han modificado notablemente la idoneidad de estos hábitats para la especie y por tanto su distribución en el sur de España (Delibes-Mateos et al. 2013b). Actualmente, donde alcanza sus máximas densidades es en las pseudo-estepas agrarias de los pisos bioclimáticos mediterráneos, con presencia de alta diversidad de cultivos (en particular la trilogía mediterránea de cereal, olivo y viñedo), entremezclados con zonas de vegetación natural (monte bajo, pastizales y barbechos) y abundantes lindes (bandas lineales de vegetación arvense o arbustiva situadas en los bordes o interior de parcelas cultivadas, Lucio y Purroy 1992, Peiró et al. 1993, Vargas et al. 2006, Casas y Viñuela
2010, Duarte 2012). Estudios de radio-seguimiento en hábitats pseudo-esteparios de Castilla-La Mancha mostraron que, en términos absolutos, el sustrato de nidificación mayoritario de la perdiz es el cereal (47\%), seguido de lindes y barbechos (22 y $12 \%$ respectivamente, Casas y Viñuela 2010). Sin embargo, la comparación entre los sustratos disponibles y los utilizados ha mostrado que el cereal es seleccionado negativamente, mientras que las lindes lo son positivamente (Casas y Viñuela 2010, Villanúa et al. 2011).

Existe una estrecha relación entre la productividad de las poblaciones de perdiz y la calidad del hábitat. La intensificación de la agricultura ha provocado un declive generalizado de las poblaciones de aves ligadas a estos medios (Green et al. 2005). El análisis de datos históricos de bolsas de caza en España indica que la concentración parcelaria, junto a la excesiva presión cinegética de poblaciones en declive explican buena parte del colapso poblacional de la perdiz (Blanco-Aguiar 2007, Blanco-Aguiar et al. manuscrito no publicado). La disponibilidad de lindes y la heterogeneidad del paisaje son dos de los factores que más afectan a la supervivencia de los adultos (Buenestado et al. 2009) y al éxito de nidificación (Casas y Viñuela 2010, Villanúa et al. 2011; Duarte 2012). Además, a pesar del efecto potencial importante de la depredación en el éxito de nidificación de la perdiz roja (Potts 1980), en hábitats pseudo-esteparios la cosecha es la principal causa de pérdida de nidos (Casas y Viñuela 2010, Villanúa et al. 2011). La adopción generalizada del uso de cereales de ciclo corto ha supuesto una cosecha más temprana y un acortamiento del periodo transcurrido entre el momento en que el cereal tiene una estructura adecuada para que la perdiz instale el nido y la cosecha. Esto ha convertido a los campos de cereal en una "trampa ecológica" para las perdices: no tienen más remedio que instalar el nido en este hábitat, dada la escasez de alternativas adecuadas, como por ejemplo las lindes, pero este es el lugar de nidificación con menor éxito (Casas y Viñuela 2010). Resultados muy similares se han encontrado en poblaciones del sur de Navarra (Villanúa et al. 2011), a pesar de las notables diferencias climáticas, de hábitat o de comunidad de depredadores que hay entre ambas zonas, lo que posiblemente convierte a este problema en uno de los más importantes para las poblaciones de perdiz roja, ya que el éxito de eclosión es la variable demográfica clave en esta especie (Ferreras et al., datos sin publicar). Similares efectos negativos de la gestión agraria moderna se ha detectado también en el olivar (Duarte 2012).

Por último, recientemente se ha demostrado que las semillas "blindadas" de cereal (semillas con una capa protectora por impregnación con fungicidas e insecticidas) causan serios problemas fisiológicos y reproductivos o incluso mortalidad en las perdices que las consumen (López-Antia et al. 2013). Queda por estudiar en condiciones de campo cual es el consumo de semillas tratadas por las perdices para evaluar sus efectos reales sobre las poblaciones silvestres. Además, el ácido nítrico usado para limpiar tubos de riego de cultivos como la vid o el olivo puede también significar un importante riesgo (Rodríguez-Estivel et al. 2010).

\section{El controvertido papel de la depredación}

La perdiz roja tiene un alto valor ecológico por su importancia como presa para gran parte de los depredadores ibéricos (Calderón 1977, Yanes et al. 1998). Aunque la depredación es uno de los factores potencialmente más influyente en la dinámica de poblaciones de especie presa (Newton 1998), los trabajos científicos que han cuantificado la incidencia de la depredación en las distintas fases del ciclo de vida de la perdiz roja y su efecto en la dinámica de poblaciones son aún escasos. Se ha encontrado que la tasa de depredación de nidos aumenta con la densidad de perdices (Herranz et al. 2002) y la de adultos cuando disminuye la densidad y/o detectabilidad de presas alternativas como el conejo (Ontiveros et al. 2005). Agrupando las mortalidades debidas a depredación en las distintas clases de edad (huevo, pollo, joven y adulto), parece que la mayor parte de la depredación se debe a carnívoros (principalmente zorros y perros asilvestrados) y córvidos (principalmente urraca Pica pica) y en menor proporción a rapaces (Yanes et al. 1998, Herranz et al. 2002, Casas y Viñuela 2005, Buenestado et 
al. 2009, Ferreras et al., datos sin publicar, Gaudioso et al 2011; Villanúa et al. 2011). La espectacular expansión reciente del jabalí puede estar convirtiendo también a esta especie en uno de los depredadores de nidos más importantes (García y Vargas 2000).

En el estudio experimental más completo realizado hasta el momento en España, Mateo-Moriones et al. (2012) evaluaron la efectividad del control selectivo de zorros y urracas, y encontraron una mejora de la supervivencia en los pollos, principalmente tras el primer mes de vida, pero no de la supervivencia de adultos y nidos. Por otro lado, otros trabajos han observado que la supervivencia de nidos de perdiz naturales o artificiales era mayor en zonas sujetas a control de depredadores (mamíferos: Ricci et al. 1990, urracas: Herranz 2000). Sin embargo, en un estudio correlativo llevado a cabo en 48 cotos de la mitad sur peninsular no se encontró ninguna relación entre la abundancia postreproductora de perdiz y la intensidad del control de depredadores (Díaz-Fernández et al., 2013), ni se ha registrado una clara asociación entre abundancia de depredadores y de perdiz roja en esta región (Herranz 2000).

En general, los estudios realizados hasta el momento no permiten concluir de forma general que haya una clara relación causaefecto entre el control de depredadores y una mayor densidad de perdices silvestres, y no parece que la depredación haya provocado un efecto a gran escala en la abundancia de poblaciones de perdiz (Blanco-Aguiar et al. 2012, pero ver Moleón et al. 2013). Por otro lado, el control de depredadores parece ser poco eficaz cuando el principal problema es la mala calidad del hábitat (Arroyo et al. 2010, Ferreras et al., datos sin publicar; Duarte 2012). Un aumento en la heterogeneidad del paisaje y una mayor densidad de lindes podrían disminuir el efecto relativo de la depredación en la demografía, reduciendo la necesidad de la utilización de control de depredadores.

\section{Repercusiones ecológicas de las sueltas de perdices de granja}

Al igual que observamos en otras galliformes cinegéticas (ver en este mismo monográfico el artículo de Puigcerver et al. 2013), la cría en cautividad y suelta en el campo de perdices de granja es una práctica habitual en la actualidad. Comenzó a desarrollarse en España a mediados del siglo XX al amparo del antiguo ICONA (Sánchez-García et al. 2009), como respuesta a la fuerte disminución poblacional de esta especie, y como técnica de refuerzo poblacional. No obstante, no fue hasta finales de los noventa cuando esta actividad alcanzó una importancia notable (Blanco-Aguiar et al. 2008). Actualmente se sueltan al menos 3-4 millones de perdices de granja al año para su caza, fomentando el desarrollo de un importante negocio alrededor de la cría, suelta y caza de aves procedentes de granja (Sánchez-García et al. 2009, Garrido 2012).

La alta productividad de la especie junto a su relativa adaptabilidad a la cautividad, facilitaron enormemente su cría en granja, llevándose a cabo programas de cría intensiva (Sánchez-García et al. 2009). Sin embargo, debido a la baja tasa de supervivencia en el campo de las aves procedentes de granja (Gortázar et al. 2000, Alonso et al. 2005, Gaudioso et al. 2011), parece dudoso que estas sueltas hayan contribuido a recuperar las poblaciones silvestres (Blanco-Aguiar et al. 2012). La depredación ha sido mencionada repetidamente como el principal problema de mortalidad en perdices de suelta, de hecho, se ha demostrado que son más sensibles que las silvestres a esta presión (Sánchez-García et al. 2011).

Existen diferencias tanto en la diversidad como la prevalencia de parásitos y bacterias entre perdices de granja y perdices silvestres (Millán et al. 2004, Millán 2009, Díaz-Sánchez et al. 2012). Debido a la falta de controles sanitarios, estos parásitos y bacterias típicos de granja se están diseminando en el campo y afectando a las poblaciones naturales de perdices (Villanúa et al. 2008, Millán 2009, Díaz-Sánchez et al. 2012). Las poblaciones silvestres suelen tener menor resistencia a parásitos con los cuales no han tenido contacto previo (Newton 1998), lo cual podría repercutir en su eficacia biológica. La última mala noticia sanitaria para las poblaciones silvestres de perdiz roja ha sido la aparición del virus bagaza, patógeno africano que ha diezmado poblaciones silvestres en el sur de España (Gamino et al. 2012) y posiblemente en otras áreas, sin que pueda descartarse un papel de las granjas en su dispersión (Agüero et al. 2011).

Por otro lado, la perdiz roja ha sido artificialmente hibridada en granja con la perdiz chukar ( $A$. chukar), para conseguir individuos más productivos. Los híbridos de segunda generación de ambas especies son fenotípicamente iguales a una perdiz roja (Negro et al. 2001) y sólo se pueden distinguir por medio de marcadores moleculares. La ausencia de controles de calidad genética en las granjas ha extendido el problema al campo, de forma que estos ejemplares híbridos se encuentran ampliamente distribuidos por la península ibérica en la actualidad, en especial en cotos donde se sueltan perdices (Blanco-Aguiar et al. 2008), pero también pueden aparecer en cotos próximos (Casas et al. 2013). Los híbridos tienen una menor supervivencia que los individuos "puros", pero son capaces de reproducirse con éxito en el campo, e incluso tienen mayor tamaño de puesta, por tanto constituyen una seria amenaza para el mantenimiento de la integridad genética de la especie (Casas et al. 2012).

Por último, la suelta de perdices puede provocar una sobre-explotación de la población silvestre si para establecer los cupos de captura se toma como base el número de perdices soltadas, ya que la mortalidad de las aves de granjas es muy alta en las primeras semanas tras la suelta (Gortázar et al. 2000).

\section{Gestión cinegética de perdiz roja}

En el centro-sur de España podemos distinguir tres modelos de gestión de perdiz roja, aplicables al resto del territorio estatal (Arroyo et al. 2012): (1) Los cotos con vocación social, que suelen ser de gran tamaño, donde un grupo de amigos o vecinos de un determinado municipio practican la caza como forma de entretenimiento, de la que no se pretende obtener rédito económico. En estos cotos, cubiertos en gran medida por terreno agrícola, otros usos del suelo, como la ganadería, son muy frecuentes. (2) Los cotos comerciales no intensivos, normalmente de menor tamaño y con mayores porcentajes de vegetación natural (no cultivada) que los sociales, en los que el gestor trata de obtener un beneficio económico de la caza. (3) Por último, los cotos intensivos, también comerciales, en los que se pueden soltar perdices de granja en número ilimitado y, según la Comunidad Autónoma, a menudo se puede practicar la caza en ellos durante periodos más largos que la temporada de caza regular. Por tanto, existe un gradiente de intensidad de gestión que va incrementándose de los cotos sociales a los cotos intensivos. El número de comederos $/ \mathrm{km}^{2}$, por ejemplo, es más de cinco veces superior en los cotos intensivos que en los comerciales no intensivos y cinco veces mayor en éstos que en los sociales (Arroyo et al. 2012). Patrones similares se observan para la densidad de bebederos, el porcentaje de terreno sembrado para la caza o el número de depredadores controlados. Por ejemplo, se controlan casi el doble de zorros en cotos intensivos que en los comerciales no intensivos y casi el triple que en los sociales, lo cual no resulta sorprendente dado que la depredación por este carnívoro es una de las mayores causas de mortalidad de las perdices de granja una vez que son liberadas en el campo (Alonso et al. 2005). No obstante, las mayores diferencias en la gestión de estos cotos se observan en la suelta de perdices. Así, en los intensivos se sueltan en promedio más de 2000 perdices $/ \mathrm{km}^{2}$, mientras que estas cifras descienden en los cotos comerciales no intensivos ( 15 por $\left.\mathrm{km}^{2}\right)$ y sobre todo en los sociales ( 1.5 por $\mathrm{km}^{2}$ ). En los cotos intensivos, además, se sueltan perdices todos los años, mientras que en los otros dos tipos de coto no se suele soltar con tanta frecuencia. Para poder llevar a cabo esta gestión intensiva, los cotos comerciales tienen un mayor número de guardas (Arroyo et al. 2012). 
La caza en ojeo, aquella en la que un grupo de batidores van dirigiendo a las perdices hacia una línea de puestos de cazadores, es mucho más habitual en los cotos intensivos que en los otros tipos de coto, en los que predomina la caza en mano. El número de cazadores por $\mathrm{km}^{2}$ y día es mucho menor en los intensivos, pero, como en estos cotos se caza durante muchos más días, la presión cinegética es similar a la de los otros tipos de cotos (Arroyo et al. 2012). Finalmente, se cazan anualmente muchísimas más perdices en cotos intensivos $\left(\sim 1270 / \mathrm{km}^{2}\right.$, máximo de más de $\left.3000 / \mathrm{km}^{2}\right)$ que en comerciales no intensivos $\left(\sim 39 / \mathrm{km}^{2}\right)$ y en éstos que en sociales $\left(\sim 18 / \mathrm{km}^{2}\right)$.

\section{¿Es la gestión cinegética actual eficaz para mantener la perdiz silvestre?}

En los cotos intensivos, la bolsa de caza de perdiz depende exclusivamente de la cantidad de perdices soltadas, lo que convierte a esta caza en una actividad "industrial" completamente desacoplada de la dinámica demográfica de la población silvestre (DíazFernández et al. 2012). En los cotos no intensivos, la bolsa de caza estaba relacionada con la abundancia estival de perdiz silvestre y el hábitat (más capturas donde hay más perdices, en cotos con más superficie ocupada por monte mediterráneo) y de la presión cinegética, pero no con la cantidad de perdices soltadas. Por tanto, las sueltas de perdices en bajo número que se realizan a menudo en cotos no intensivos no parecen ser eficaces ni siquiera para aumentar las bolsas de caza, que dependen más de la abundancia de perdiz silvestre.

Se ha evaluado recientemente la relación entre determinadas medidas de gestión cinegética y la abundancia de perdiz en cotos del centro peninsular, usando datos de censos y encuestas a los gestores de los cotos (Díaz-Fernández et al. 2013). La abundancia de perdiz se estimó en verano, después de la cosecha y antes de las sueltas de perdiz de granja (que suelen ocurrir cerca del inicio de la temporada cinegética); esta variable es un buen indicador de la producción de pollos y de la disponibilidad de ejemplares silvestres para la caza en otoño.

En los cotos no intensivos, y teniendo en cuenta las diferencias de hábitat entre cotos, la abundancia de perdices aumentaba con la densidad de comederos y bebederos, pero disminuía con la cantidad de aves de granja liberadas y la presión de caza. No se encontró, en cambio, ningún efecto claro del control de depredadores. Por tanto, la suelta de perdices en pequeño número no solo no es favorable para aumentar la densidad de perdiz silvestre, sino que incluso está asociada a una menor productividad de las poblaciones. El efecto positivo de comederos y bebederos sugiere que ésta es una práctica de gestión beneficiosa para las poblaciones silvestres, pero también puede indicar que el medio natural en que están viviendo las perdices tiene problemas de disponibilidad de alimento y agua.

Por último, el ajuste anual de la presión cinegética a la abundancia es un elemento clave de cualquier sistema sensato de gestión sostenible (Lucio 1998), y el estudio previo muestra que la abundancia de perdiz es menor en cotos con un mal ajuste. Sería necesario por tanto realizar mejores estimas de la abundancia como tener mecanismos más eficaces de regulación de las capturas.

\section{Aspectos socio-económicos de la caza de la perdiz}

La perdiz roja genera una gran actividad social y económica en España, donde caza alrededor del $3 \%$ de la población española y 70000 cazadores extranjeros anualmente (Reginfo 2008). Las cacerías de perdiz son particularmente importantes en el centro-sur peninsular, pero se caza perdiz roja en prácticamente todas las regiones (MARM 2006), por lo que los efectos sociales y económicos de esta actividad llegan al medio rural de casi todo el país. Se ha estimado el movimiento económico generado por la caza de perdiz roja en España en más de 1150 millones de $€$ anuales, más del $25 \%$ del total generado por la caza en conjunto (Garrido 2012). En España el $88 \%$ del área acotada se gestiona de forma privada (MARM 2006), con posibilidad de comercialización en muchos de los casos. No obstante, no se conoce de forma precisa el número de cotos comerciales que hay en nuestro país. Dado que la gestión es más intensa en cotos comerciales, en ellos se generan más costes y más beneficios para terceros, incluyendo más puestos de trabajo, en particular en los cotos intensivos (Arroyo et al. 2012). Recientemente se ha visto que los principales beneficiarios indirectos de los costes de la gestión en los cotos de perdiz roja son la mano de obra local, aunque los productores de trigo y piensos y los de perdiz de granja en el caso de cotos intensivos, se llevan también una parte importante de estos beneficios (Díaz-Fernández 2012). Por último, el estado es también un beneficiario indirecto. Por ejemplo, el gobierno regional de Castilla La Mancha recaudó más de 2 millones de euros en 1996 por gastos legales directamente relacionados con la caza (excluyendo los impuestos pagados directamente por los cotos) (Bernabéu 2002).

La caza de perdiz roja es una actividad (económica o no) complementaria a otras en muchas fincas (Bernabéu 2002, Arroyo et al. 2012). Esto puede tener varias implicaciones. Por un lado, la caza puede generar beneficios complementarios a los producidos por otros recursos en sistemas multifuncionales (Bernabéu 2002). Por otro, al ser una actividad secundaria, puede que la dedicación a optimizar la gestión de esta actividad sea baja, llevando a una gestión ineficiente para la caza. De hecho, un estudio reciente indica que las sueltas de perdiz de granja en cotos no intensivos no mejoran la rentabilidad de los mismos, pero sí la de los intensivos, a pesar de sus mayores costes anuales (Díaz-Fernández 2012), lo que explica el interés reciente en su desarrollo. No obstante, la mayor rentabilidad de los cotos intensivos frente a los que gestionan perdiz silvestre, cuando ambos modelos compiten en el mismo mercado al mismo precio, se debe también a la ausencia de internalización de los costes ecológicos de las sueltas (Díaz-Fernández 2012). El estudio indicaba, asimismo, que los cotos no intensivos sin sueltas, particularmente en escenarios de precios medios a altos, pueden ser negocios rentables, aunque sus beneficios no sean comparables con los de los cotos intensivos. Para aumentar los beneficios que supone la caza en cotos no intensivos, las inversiones deberían re-direccionarse hacia la mejora de la eficiencia técnica de la gestión de las poblaciones silvestres y a diferenciar claramente la calidad ecológica del producto en el mercado.

\section{Conclusiones}

Los fuertes cambios a gran escala ocurridos en los modelos de gestión agraria parecen estar detrás tanto del declive histórico de las poblaciones de perdiz como de la mala situación actual de sus poblaciones silvestres. La Política Agraria Europea (PAC) podría ser la herramienta clave para re-direccionar la gestión agraria hacia prácticas que permitan mejorar, tanto la situación crítica de muchas especies amenazadas asociadas a ecosistemas agrarios, como la de especies cinegéticas con importancia socio-económica, como la perdiz roja. Para este caso concreto, promover la presencia de lindes sería una de las acciones más relevantes.

Las sueltas de perdices de granja están poniendo en riesgo la integridad genética de la especie, no mejoran las bolsas de caza cuando se sueltan perdices en bajo número, y parecen afectar negativamente a la productividad de las poblaciones silvestres. En definitiva, las perdices de granja han permitido mantener buena parte de la actividad cinegética, pero no están contribuyendo a una recuperación eficaz de las poblaciones silvestres, más bien al contrario. Por lo tanto, su uso debería prohibirse (como en Navarra) o al menos regularse de forma mucho más eficaz. Sería importante, igualmente, que las perdices de granja apareciesen identificadas como tal (sistemas obligatorios y seguros de marcaje que permitan la trazabilidad del producto) y la aplicación de protocolos de control genético y sanitario eficientes. 
Se necesita más información sobre el efecto de la depredación y el control de depredadores sobre la producción de perdiz roja silvestre, ya que los resultados disponibles no son concluyentes, pero globalmente no parecen justificados, ni la creencia popular sobre la alta relevancia de este factor, ni el uso generalizado de esta herramienta de gestión cinegética.

En conclusión, existe abundante información científica que apunta a que la alternativa para la gestión de esta especie más beneficiosa para la conservación de la población silvestre estaría basada en el ajuste anual de la presión cinegética a la abundancia de perdices, un buen manejo del hábitat y la suplementación de agua y comida, limitando en cambio la suelta de animales de granja. Este modelo de gestión podría ser favorable para otras especies no cinegéticas (Arroyo et al. 2013, este número). Si existiera una buena identificación en el mercado de los cotos que realizan una gestión sostenible de las poblaciones silvestres de perdiz, esto podría aumentar los beneficios que supone la caza en dichos cotos, promocionando oficialmente una gestión favorable tanto para aprovechar un importante recurso natural renovable, como para la conservación del patrimonio natural español.

Por último, teniendo en cuenta las circunstancias, no parece descabellado plantear la necesidad de revisar el status real oficial de esta especie, aunque se estén cazando millones de ejemplares al año.

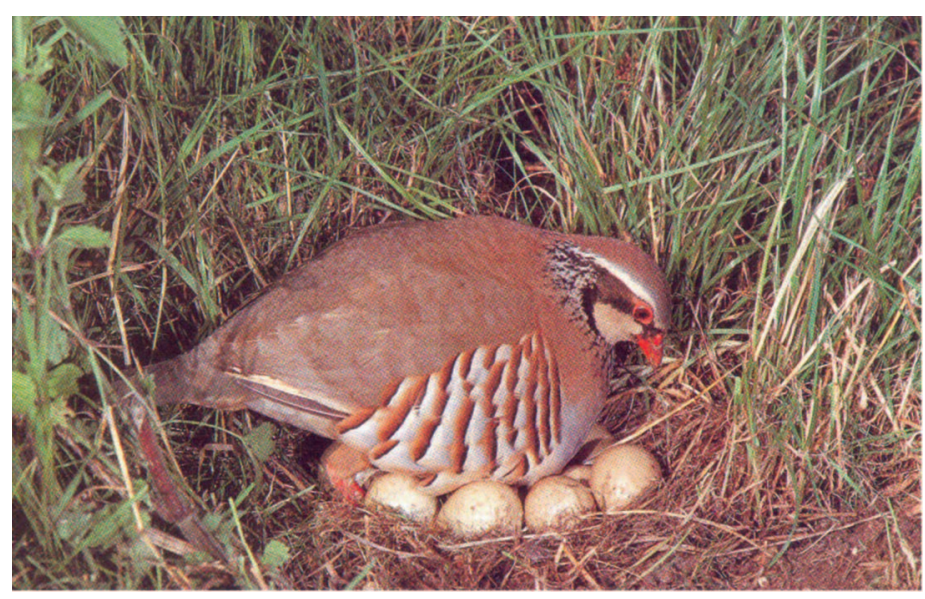

Figura 1. La perdiz roja tiene un peculiar sistema de reproducción basado en la doble puesta. Alrededor del 50 \% de las hembras en años climatológicamente favorables ponen huevos en dos nidos, uno incubado por el macho y otro por ella misma (Casas et al. 2009). El éxito de eclosión es por tanto una variable particularmente importante en la demografía de esta especie.
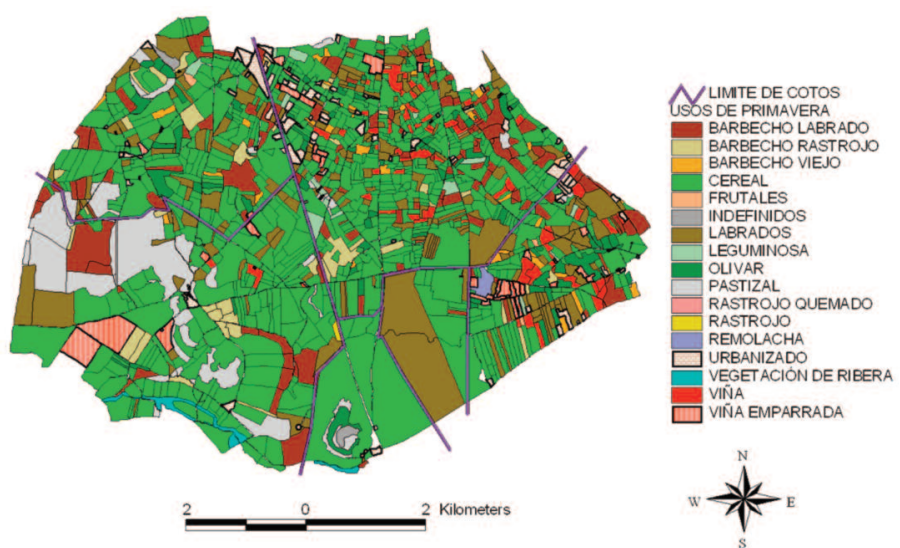

Figura 2. Área de estudio con hábitat agrario cercana a Ciudad Real, donde se han desarrollado estudios de radio-seguimiento. El paisaje en primavera está claramente dominado por el cereal, que se ha convertido en el principal hábitat de nidificación de la especie por la escasez de otras alternativas. Pero este es uno de los hábitats con menor éxito reproductivo, principalmente por las pérdidas causadas durante la cosecha. El cereal se ha convertido en una trampa ecológica para esta especie (Casas y Viñuela 2010).

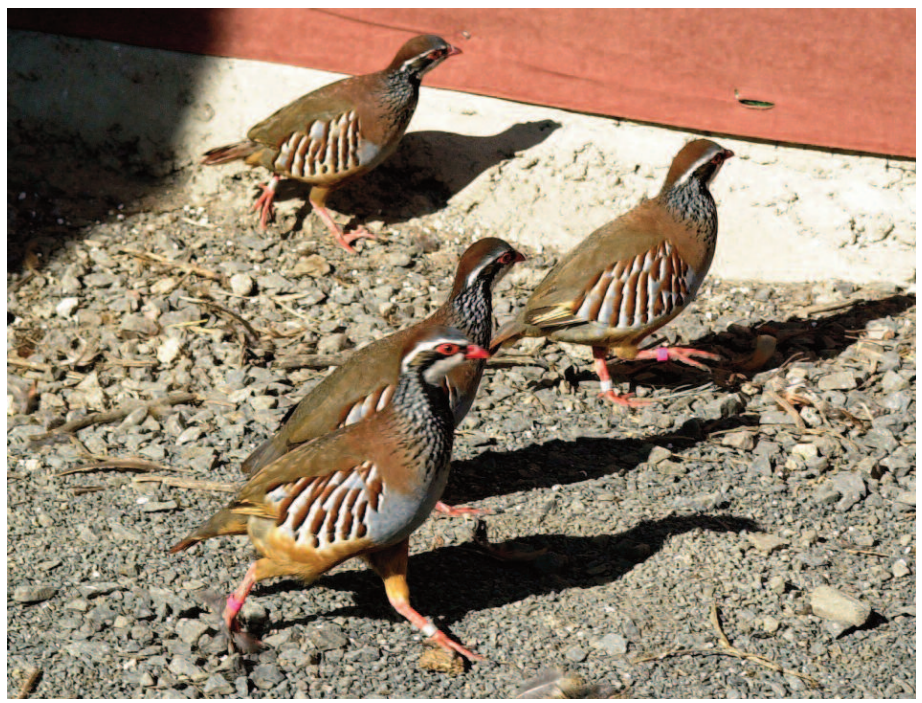

Figura 3. La suelta de perdices antes o durante la temporada de caza se ha convertido en una práctica habitual, con una industria detrás, ya que se liberan al menos 3-4 millones de perdices al año. Sin embargo, esta práctica no ha ayudado a la recuperación de las poblaciones silvestres, sino que se ha convertido en un problema añadido por contaminación genética, transmisión de parásitos y enfermedades, disminución del éxito reproductivo de las poblaciones que reciben las perdices soltadas y sobrecaza de ejemplares silvestres.

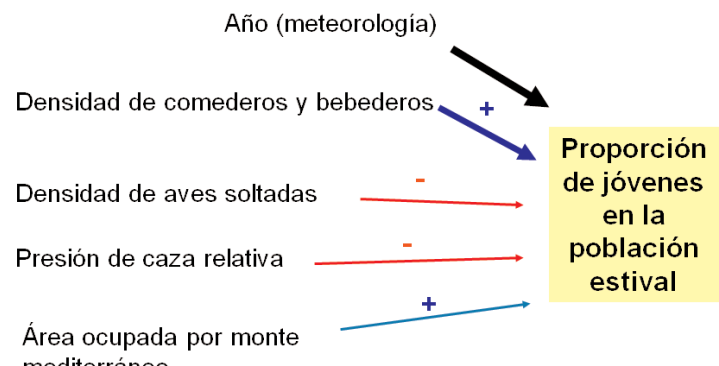
mediterráneo

Densidad de zoxros y trracas eliminadas con control de depredadores

Figura 4. Factores que afectan a la proporción de jóvenes en las poblaciones estivales de perdiz roja en una amplia muestra de cotos del centro de España (Madrid, Castilla-La Mancha y Extremadura) (Díaz-Fernández et al. 2013). El grosor de las flechas indica la importancia relativa de cada efecto. La presión de caza relativa son los residuos de la regresión entre densidad de perdices cazadas y densidad estival de perdices (es decir, si se cazan más o menos de lo esperable por la abundancia de perdiz en el coto). La proporción de jóvenes en la población estival es un buen indicador de la productividad de pollos, y por tanto de la disponibilidad de perdices para la caza en otoño e invierno.

\section{Referencias}

Aebischer, N.J., Potts, G.R. 1994. Red-legged partridge. En: Tucker, G.M., Heath, M.F. (eds.), Birds in Europe. Their conservation status. Birdlife Conservation Series No. 3, Birdlife International, Cambridge, UK.

Agüero, M., Fernandez-Piñero, J., Buitrago, D., Sanchez, A., Elizalde, M., San Miguel, E., Villalba, R., Llorente, F., Jimenez-Clavero, M.A. 2011. Bagaza virus in partridges and pheasants, Spain, 2010. Emerging Infectious Diseases 17:1498-1501.

Alonso, M.E., Pérez, J.A., Gaudioso, V.R., Diéz, C., Prieto, R. 2005. Study of survival, dispersal and home range of autumn-released red-legged partridges (Alectoris rufa). British Poultry Science 46:401-406.

Arroyo, B., Delibes-Mateos, M., Blanco-Aguiar, J.A., Vargas, J.M. 2010. Contribución de la agricultura al aprovechamiento sostenible de la caza menor. En: Moyano, E. (Ed.). Agricultura familiar en España. Anuario 2010. Pp. 174-179. Fundación de Estudios Rurales, Madrid, España. 
Arroyo, B., Delibes-Mateos, M., Díaz-Fernández, S., Viñuela, J. 2012. Hunting management in relation to profitability aims: red-legged partridge hunting in central Spain. European Journal of Wildlife Research 58:433-439.

Arroyo, B., Delibes-Mateos, M., Caro, J., Estrada, A., Mougeot, F., Díaz-Fernández, S., Casas, F., Viñuela, J. 2013. Efecto de la gestión para las especies de caza menor sobre la fauna no cinegética. Ecosistemas 22(2):27-32.

Bernabéu, R. 2002. La caza en Castilla La Mancha y sus estrategias de desarrollo. Tesis Doctoral, Universidad de Castilla La Mancha. Cuenca, España.

Blanco-Aguiar, J.A. 2007. Variación espacial en la biología de la perdiz roja (Alectoris rufa): una aproximación multidisciplinar. Tesis Doctoral, Universidad Complutense de Madrid, España.

Blanco-Aguiar, J.A., Virgós, E., Villafuerte, R. 2003. Perdiz Roja (Alectoris rufa). En: Martí, R., Del Moral, J.C. (eds.), Atlas de las aves reproductoras de España, pp. 212-213. Dirección General de Conservación de la Naturaleza y Sociedad Española de Ornitología, Madrid, España.

Blanco-Aguiar, J.A., González-Jara, P., Ferrero, E., Sánchez-Barbudo, I., Virgós, E., Villafuerte, R., Dávila, J.A. 2008, Assessment of game restocking contributions to anthropogenic hybridization: the case of the Iberian red-legged partridge. Animal Conservation 11:535-545.

Blanco-Aguiar, J.A., Delibes-Mateos, M., Arroyo, B., Ferreras, P., Casas, F., Real, R., Vargas, M.J., Villafuerte, R., Viñuela, J. 2012. Is the interaction between rabbit hemorrhagic disease and hyperpredation by raptors a major cause of the red-legged partridge decline in Spain? European Journal of Wildlife Research 54:433-439.

Buenestado, F.J., Ferreras, P., Blanco-Aguiar, J.A., Tortosa, F.S., Villafuerte, R. 2009. Survival and causes of mortality among wild Red-legged Partridges Alectoris rufa in southern Spain: Implications for conservation. Ibis 151:720-730.

Calderón, J. 1977. El papel de la perdiz roja (Alectoris rufa) en la dieta de los predadores ibéricos. Doñana, Acta Vertebrata 4:61-126.

Casas F., Viñuela, J. 2005. Mortality rate differences in two populations of red-legged partridge (Alectoris rufa) subject to different game management systems. Extended abtract book of the XXVII Congress of the International Union of Game Biologist (UIGB), pp. 306. 28 August - 3 September 2005, Hannover, Germany.

Casas, F., Viñuela, J. 2010. Agricultural practices or game management: which is the key to improve red-legged partridge nesting success in agricultural landscapes? Environmental Conservation 37:177-186.

Casas, F., Mougeot, F., Viñuela, J. 2009. Double nesting behaviour and differences between sexes in breeding success in wild red-legged partridges Alectoris rufa. Ibis 151:743-751.

Casas F., Mougeot F., Ferrero, M.E., Sanchéz-Barbudo I., Dávila J.A., Viñuela J. 2012. Fitness consequences of anthropogenic hybridization in wild red-legged partridge (Alectoris rufa, Phasianidae) populations. Biological Invasions 14:295-305.

Casas, F., Mougeot, F., Sanchéz-Barbudo, I., Dávila, J.A., Viñuela, J. 2013 Phenotypic differences in body size, body condition and circulating carotenoids between hybrid and "pure" red-legged partridges (Alectoris rufa) in the wild. Journal of Ornithology 154:803-811.

Delibes-Mateos, M., Díaz-Fernández, S., Ferreras, P., Viñuela, J., Arroyo, B. 2013a. The role of economic and social factors driving predator control in small game estates in central Spain. Ecology and Society 18(2): 28.

Delibes-Mateos, M., Farfán, M.A., Olivero, J, Vargas, J.M. 2013b. Impact of land-use changes on red-legged partridge conservation in the Iberian Peninsula. Environmental Conservation 39:337-346.

Díaz-Fernández, S. 2012. Relaciones entre la gestión cinegética de perdiz roja, las poblaciones de perdiz roja y las poblaciones humanas. Tesis doctoral. Universidad de Castilla La Mancha, Ciudad Real, España.

Díaz-Fernández, S., Viñuela, J. Arroyo, B. 2012. Harvest of red-legged partridge in central Spain. Journal of Wildlife Management 76:1354-1363.

Díaz-Fernández, S., Arroyo, B., Casas, F., Martínez-Haro, M., Viñuela, J. En prensa. Effect of game management on red-legged partridge abundance. Plos One 8(6):e66671.

Díaz-Sánchez, S., Mateo Moriones, A., Casas, F., Höfle, U. 2012. Prevalence of Escherichia coli, Salmonella sp. and Campylobacter sp. in the intestinal flora of farm-reared, restocked and wild red-legged partridges (Alectoris rufa): is restocking using farm-reared birds a risk? European Journal of Wildlife Research 58:99-105.

Duarte, J. 2012. Ciclo reproductor y aprovechamiento cinegético de la perdiz roja (Alectoris rufa) en Andalucía. Tesis Doctoral, Universidad de Málaga, España.
Gamino, V., Gutierrez-Guzmán, A.V., Fernández de Mera, I.G., Ortiz, J.A., Durán-Martína, M., de la Fuente J., Gortázar C., Hofle U. 2012. Natural bagaza virus infection in game birds in southern Spain. Veterinary Research 43:65.

García, F.J. Vargas, J.M. 2000. Jabalíes, los mayores depredadores de nidos. Trofeo 360:46-50.

Garrido, J.L. 2012. La caza. Sector económico. Valoración por subsectores. FEDENCA-EEC, Madrid, España.

Gaudioso, V.R., Sánchez-García, C., Pérez, J.A., Rodríguez, P.L., Armenteros J.A., Alonso M.E. 2011. Does early antipredator training increase the suitability of captive red-legged partridges (Alectoris rufa) for releasing? Poultry Science 90:1900-1908

Gortázar, C., Villafuerte, R., Martín, M. 2000. Success of traditional restocking of red-legged partridge for hunting purposes in areas of low density of northeast Spain Aragón. Zeitschrift fur Jagdwissenschaft 46:23-30.

Green, R.E. 1984. Double nesting of the red-legged partridge Alectoris rufa. Ibis 126:332-334.

Green, R.E., Cornell, S.J., Scharlemann, J.P.W., Balmford, A. 2005. Farming and the fate of wild nature. Science 307:550-555.

Herranz, J. 2000. Efectos de la depredación y del control de depredadores sobre la caza menor en Castilla-La Mancha. Tesis doctoral, Universidad Autónoma de Madrid, España.

Herranz, J., Yanes, M., Suárez, F. 2002. El impacto de la predación sobre las poblaciones de perdiz roja. En: Lucio, A., Sáenz de Buruaga, M. (eds.), Aportaciones a la gestión sostenible de la caza, pp. 81-100. FEDENCA-EEC, Madrid, España.

Lopez-Antia, A., Ortiz-Santaliestra, M.E., Mougeot, F, Mateo, R. 2013. Experimental exposure of red-legged partridges (Alectoris rufa) to seeds coated with imidacloprid, thiram and difenoconazole. Ecotoxicology 22:125-138.

Lucio, A.J. 1990. Influencia de las condiciones climáticas en la productividad de la perdiz roja (Alectoris rufa). Ardeola 37:207-218.

Lucio, A.J. 1998. Recuperación y gestión de la perdiz roja en España. En: Sáenz de Buruaga, M. (coord.), La Perdiz Roja. I curso, pp. 63-92. Grupo Editorial V-FEDENCA. Madrid, España.

Lucio, A.J., Purroy, F.J. 1992. Red-legged partridge (Alectoris rufa) habitat selection in Northwest Spain. Gibier Faune Sauvage 9:417-430.

Mateo-Moriones, A., Villafuerte, R., Ferreras, P. 2012. Does fox control improve red-legged partridge (Alectoris rufa) survival? An experimental study in Northern Spain. Animal Biodiversity and Conservation 35:395-404.

Ministerio de Medio Ambiente y Medio Rural y Marino [MARM] 2006. Anuario de estadística forestal. MARM. Madrid. España.

Millán, J. 2009. Diseases of the red-legged partridge (Alectoris rufa I.): A review. Wildlife Biology in Practice 5:70-88.

Millán, J., Gortázar, C., Villafuerte, R. 2004. A comparison of the helminth faunas of wild and farm-reared red-legged partridges. Journal of Wildlife Management 68:701-707.

Moleón, M., Almaraz, P., Sánchez-Zapata, J.A. 2013. Inferring ecological mechanisms from hunting bag data in wildlife management: a reply to Blanco-Aguiar et al. (2012). European Journal of Wildlife Research 59:599-608.

Negro, J.J., Torres, M.J., Godoy, J.A. 2001. RAPD analysis for detection and eradication of hybrid partridges (Alectoris rufa x A. graeca) in Spain. Biological Conservation 98:19-24.

Newton, I. 1998. Population limitation in birds. Academic Press, San Diego. EE.UU.

Ontiveros, D., Caro, J., Pleguezuelos, J.M. 2005. Prey density, prey detectability and food habits: the case of Bonelli's eagle and the conservation measures. Biological Conservation 123:19-25.

Peiró, V., Seva, E., Almiñana, N. 1993. Selección de hábitat en una población de perdiz roja (Alectoris rufa) en una zona de sierra del sur de la provincia de Alicante. Mediterránea Serie de Biología 14:5-22.

Potts, G.R. 1980. The effects of modern agriculture, nest predation and game management on the population ecology of partridges (Perdix perdix and Alectoris rufa). Ecological Research 2:2-79.

Puigcerver, M., Sánchez-Donoso, I., Vilà, C., Sardà-Palomera, F., MoralesRodríguez, P.A., Caballero de la Calle, J.R., Rodríguez-Teijeiro, J.D. 2013. Hibridación entre la codorniz común (Coturnix coturnix) y la codorniz de granja: estado de un problema de conservación. Ecosistemas 22(2):48-53.

Reginfo, J.I. 2008. Un segmento del turismo internacional en auge: el turismo de caza. Cuadernos de Turismo 22:187-210. 
Ricci J.C., Mathon J.F., Garcia A., Berger F., Esteve J.P. (1990) . Effect of habitat structure and nest site selection on nest predation in red-legged partridges (Alectoris rufa L.) in french mediterranean farmlands. Gibier Faune Sauvage $7: 231-253$.

Rodríguez-Estivel, J., Martínez-Haro, M., Martín-Hernando, M.P., Mateo, R. 2010. Sub-chronic effects of nitrate in drinking water on red-legged partridge (Alectoris rufa): Oxidative stress and T-cell mediated immune function. Environmental Research 110:469-475.

Sánchez-García, C., Alonso, M.E., Prieto, R., González, V., Gaudioso, V.R. 2009. Una visión sobre la avicultura para la producción de caza en España. ITEA-Animal 105:169-183.

Sanchez-Garcia, C., Alonso, M.E.; Perez, J.A., Rodriguez, P.L., Gaudioso, V.R. 2011. Comparing fostering success between wild-caught and game farm bred captive red-legged partridges (Alectoris rufa, L.). Applied Animal Behaviour Science 133:70-77.

SEO/Birdlife 2013. Resultados del programa SACRE de SEO/Birdlife. SEO/Birdlife, Madrid, España.
Vargas, J.M., Guerrero, J.C., Farfán, M.A., Barbosa, A.M., Real, R. 2006. Land use and environmental factors affecting Red-legged Partridge (Alectoris rufa) hunting yields in southern Spain. European Journal of Wildlife Research 52:188-195.

Villanúa, D., Pérez-Rodríguez, L., Casas, F., Alzaga, V., Acevedo, P., Viñuela, J., Gortázar C. 2008. Sanitary risks of red-legged partridge releases: introduction of parasites. European Journal of Wildlife Research 54:199-204.

Villanúa, D., Torres, J., Ardaiz, J., Alzaga, V., Ros, F., Cormenzana, A., Castién, E. 2011. Relationship between landscape heterogeneity loss and redlegged partridge (Alectoris rufa) populations survival. Abstract book of the $X X X$ th Congress of the International Union of Game Biologist (UIGB) and PERDIX XIII. 5-9 septiembre 2011, Barcelona, España. Pp. 176.

Yanes, M., Herranz, J., de la Puente, J., Suárez, F. 1998. La Perdiz Roja. Identidad de los depredadores e intensidad de la depredación. En: Sáenz de Buruaga, M. (coord.), La Perdiz Roja. I curso, pp. 135-147. Grupo Editorial V-FEDENCA. Madrid, España. 